\title{
THE IONIZATION STATE ALONG THE BEAM OF HERBIG-HARO JETS
}

\author{
FRANCESCA BACCIOTTI \\ Dipartimento di Astronomia e Scienza dello Spazio, \\ Università di Firenze, \\ Largo E. Fermi 5, 50125 Firenze, Italy
}

\begin{abstract}
An original spectroscopic diagnostic technique has been recently developed that allows to estimate in a model-independent way the ionization fraction $x_{e}$ and the average excitation temperature in the beam of Herbig-Haro jets (Bacciotti, Chiuderi and Oliva 1995). The procedure is based on the fact that in the low excitation conditions present in this region the ionization state of oxygen and nitrogen can be assumed to be regulated by charge exchange with atomic hydrogen. The application of this technique to long-slit spectra of several well-known stellar jets indicates that the hydrogen ionization fraction $x_{e}$ starts from $0.2-0.4$ at the beginning of the flow and gently decreases along the whole jet or along sections of it as a result of time-dependent recombination. The average temperature stays almost constant, ranging from 4500 to $7000 \mathrm{~K}$. The momentum transfer rates evaluated with the derived total number densities $\left(n_{H} \sim 10^{3}-10^{4}\right.$ $\mathrm{cm}^{-3}$ ) give support to the picture in which the jet is responsible for the acceleration of a surrounding molecular outflow.
\end{abstract}

\section{Introduction}

Highly collimated, supersonic Herbig-Haro $(\mathrm{HH})$ jets emanating from young stellar objects have been the subject of intense investigations in the recent past. Many of their features, however, remain largely unexplained. Still under debate are, for example, the jet acceleration mechanism (see, e.g., Königl and Ruden 1993 for a review), the excitation of the gas in the beam (Raga and Kofman 1992, Stone and Norman 1993, Bodo et al. 1994, Ray et al. 1996), and the capability of the narrow central jet to drive a coaxial molecular outflow (see, e.g., Chernin and Masson 1995, Cabrit, this volume) 
A crucial physical parameter for any jet model is the total density of the flow which, however, is poorly known for $\mathrm{HH}$ jets. In fact, contrary to the electron density, which is easily found from the ratio of the [SII] lines at $6716 \AA$ and [SII] $6731 \AA$ (see, e.g. Osterbrock 1989), no standard procedure exists to deduce directly from observations the total hydrogen density. Its estimate has been most often derived from shock models (see, e.g., Hartigan, Morse and Raymond 1994). The results obtained this way are, however, largely model-dependent, being greatly influenced by different pre-shock conditions, by (unknown) magnetic fields, and by the assumed shape of the shock front. Alternatively, the average total density can be estimated from the luminosity in one forbidden line (Edwards et al. 1987, Cabrit et al. 1990), but this kind of analysis is severely hampered by the limited knowledge of the ionization and excitation state of the emitting species and by strong dust absorption.

Recently Bacciotti, Chiuderi and Oliva (1995, hereafter BCO95) developed an original spectroscopic diagnostic technique that allows to find in a model-independent way the ionization fraction $x_{e}=n_{H^{+}} / n_{H} \approx n_{e} / n_{H}$ of the jet gas, and hence the total density, together with the average excitation temperature. The main features of the procedure, which uses ratios of the most frequently observed forbidden lines together with $\mathrm{H} \alpha$, will be briefly recalled in the following. The results obtained from the application of the technique to several well-known $\mathrm{HH}$ jets will be reviewed, including results from spatially resolved analyses. Some interesting physical implications will be discussed.

\section{The diagnostic procedure}

The most often observed lines in the beams of $\mathrm{HH}$ jets are $\mathrm{H} \alpha$, the [SII] doublet at $6716,6731 \AA$, the [OI] $\lambda \lambda 6300,6363$ and the [NII] $\lambda \lambda 6548,6584$ lines. Here, as in all low-excitation nebulae, sulphur can be assumed to be all singly ionized. In addition, the lack of any observational evidence for high electron temperature and of any local or nearby source of energetic radiation suggests that charge exchange may be the dominant process controlling the ionization of $\mathrm{O}$ and $\mathrm{N}$. Checking this hypothesis against competing effects, as, for example, radiative plus dielectronic recombination, one finds that for oxygen charge exchange dominates unless the plasma is very close to complete ionization, while for nitrogen the charge exchange hypothesis is valid as long as $x_{e}$ is lower than about 0.5 . Regions of higher ionization are identified by the technique, but can only be described on a qualitative basis. The charge exchange hypothesis allows to express the populations of neutral $\mathrm{O}$ and singly ionized $\mathrm{N}$ as a function of $x_{e}$. Once a set of relative abundances is assumed, and the electron density is determined from standard methods 


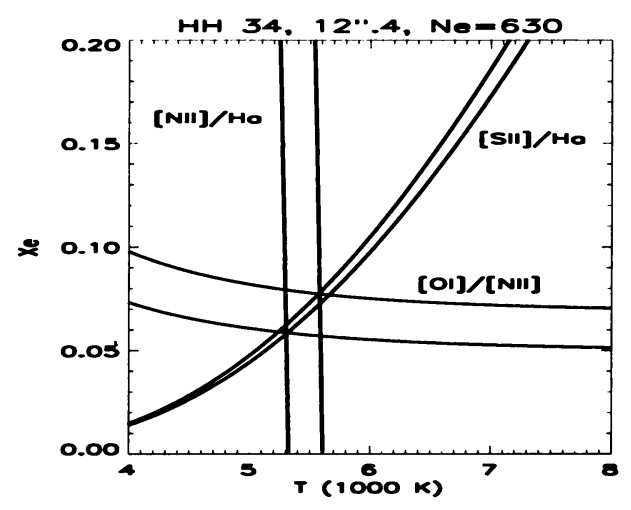

Figure 1. Example of a diagnostic diagram calculated for the position at $12 . " 4$ in the HH 34 jet. The intersection of the crossing stripes provides $T=5450 \pm 150 \mathrm{~K}$ and $x_{e}=0.067 \pm 0.008$.

any line ratio can be regarded as a known function of $x_{e}, T$, and can be calculated numerically (for more details see BCO95). A diagnostic $\left(x_{e}, T\right)$ diagram like the one in Fig. 1 provides then the required values: the strips are the loci of the $\left(x_{e}, T\right)$ values for which the predicted line ratios equal the observed ones within the measurement errors, and their intersections define the local $\left(x_{e}, T\right)$ and their uncertainty.

\section{First inspection of the HH 34 and $\mathrm{HH} 111$ jets}

As a first application BCO95 analyzed the physical conditions in the beams of the HH 34 and HH 111 jets, using spectra integrated over the most luminous knots (Reipurth et al. 1986, Reipurth 1989). Both jets turned out to be remarkably neutral: the diagnostic provided $x_{e}=0.067 \pm 0.01$ in the HH 34 beam and $x_{e}=0.11 \pm 0.02$ for the HH 111 jet. The electron density and excitation temperatures were found to be $n_{e}=720 \mathrm{~cm}^{-3}$ and $T_{e}=5800 \pm 200 \mathrm{~K}$ in the HH 34 jet, and $n_{e}=900 \mathrm{~cm}^{-3}$ and $T_{e}=5700 \pm 200 \mathrm{~K}$ in HH 111 . Combining $n_{e}$ and $x_{e}$ an average total hydrogen density of about $10^{4}$ $\mathrm{cm}^{-3}$ was found for both flows, from which the authors derived a momentum transfer rate $\dot{P} \approx 6.710^{-5} \mathrm{M}_{\odot} \mathrm{yr}^{-1} \mathrm{~km} \mathrm{~s}^{-1}$ for the HH 34 jet, and $\dot{P}=1.310^{-4} \mathrm{M}_{\odot} \mathrm{yr}^{-1} \mathrm{~km} \mathrm{~s}^{-1}$ for $\mathrm{HH} 111$.

The origin of the observed ionization degree is uncertain, however, since the low excitation of these jets can only arise from low velocity shocks $\left(v_{s}=25-35 \mathrm{~km} \mathrm{~s}^{-1}\right)$ that are not capable of producing in situ an ionization degree larger than a few per cent. On the other hand BCO95 pointed out that the typical recombination time of the jet gas is of the same order of the travel time of the bright visible section. Therefore the observed ioniza- 


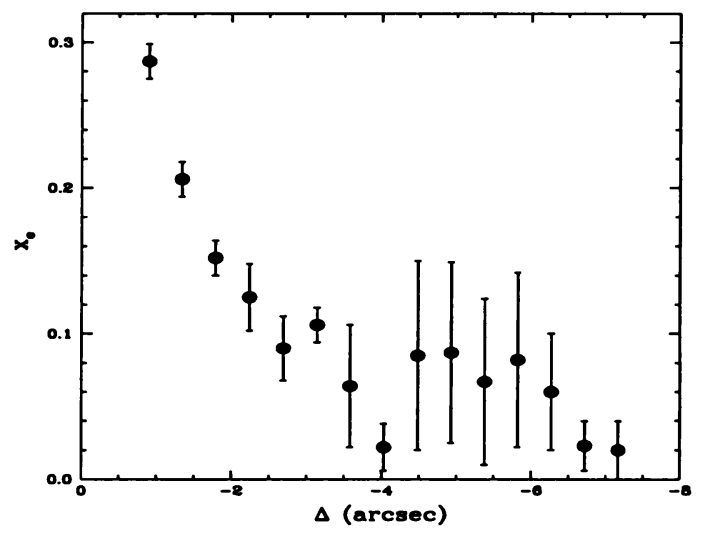

Figure 2. Ionization fraction along the axis of the optical outflow of RW Aurigae (red lobe) as a function of the distance from the star

tion state may be a remnant of the initial heating and the excitation occured in the acceleration region (for example by means of a strong steady shock heavily shielded from view by a dense circumstellar environment). Traveling away from the star the flow is subject to a sudden expansion (Mundt, Ray and Raga 1991), that 'freezes' the ionization of the gas; the jet then recollimates, but recombination is sufficiently slow to leave the gas considerably ionized also at large distances from the source. According to this scenario, $x_{e}$ should decrease along the jet axis on spatial scales determined by the product of the recombination time and the flow velocity. This prediction is not affected by the presence of weak shocks that eventually form in the beam, if these are so weak to poorly contribute to ionization.

\section{Analyses from long-slit spectra}

The observational confirmation of the picture outlined in the previous section can come from the application of the $\mathrm{BCO} 95$ procedure to spectra spatially resolved along the jet axis.

The optical jet of $R W$ Aur. As a first attempt in this direction Bacciotti, Hirth \& Natta (1996) have examined the optical bipolar outflow associated with the T Tauri star RW Aurigae, for which long-slit spectra were obtained in November 1993 with the Cassegrain twin spectrograph at the 3.5-m telescope of the Calar Alto Observatory in Spain. The results for the red lobe of the flow, in which the lines were sufficiently intense to allow a 


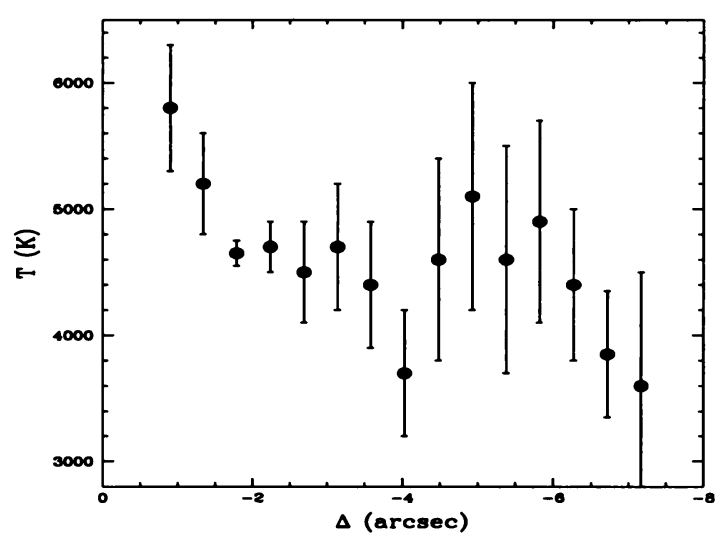

Figure 3. Average excitation temperature along the axis of the optical outflow of RW Aurigae (red lobe) as a function of the distance from the star

realistic determination, nicely confirm the suggestion offered in BCO95. As shown in Fig. 2, the ionization fraction decreases along the beam from $x_{e} \sim$ 0.28 near the star to about 0.02 at a distance of $6-7^{\prime \prime}(\sim 1000 \mathrm{AU})$, while the temperature shows a slight decline with typical values of $4500 \mathrm{~K}$ (Fig. 3). The electron density (not shown) also decreases on average along the flow, following a well-defined recombination law. The derived total density is again of the orcier of $10^{4} \mathrm{~cm}^{-3}$ which yields a mass loss rate $\dot{M} \sim 510^{-8}$ $\mathrm{M}_{\odot} \mathrm{yr}^{-1}$ and an average momentum transfer rate $\dot{P}=6.510^{-6} \mathrm{M}_{\odot} \mathrm{yr}^{-1}$ $\mathrm{km} \mathrm{s}^{-1}$.

The short RW Aur outflow, however, belongs to a system more evolved than the sources of "classical" Herbig-Haro jets. It is therefore of great interest to further investigate the cases of prototypical jets in order to obtain a model-independent determination of the fundamental physical parameters for each of them, and to establish if the agreement between the BCO95 model and the observed trends is confirmed on a large statistical basis. Recent analyses of long-slit spectra of a sample of well-known jets are illustrated in Bacciotti and Eislöffel (1997, hereafter BE97). Here we report on the results obtained for the $\mathrm{HH} 34$ and the $\mathrm{HH} 24 \mathrm{C} / \mathrm{E}$ jets as representative cases (for a detailed description of the objects see, e.g., Eislöffel and Mundt 1992, Mundt, Ray and Raga 1991). The long-slit spectra were taken in February 1987 at the ESO/MPI 2.2m-telescope on La Silla using the Boller \& Chivens spectrograph. The spectral resolution was $1.6 \AA$ per pixel and the spatial resolution $1 .^{\prime \prime} 78$ per pixel along the slit. The results are given in Figures 4,5 and 6 in the form of columns of graphs, illus- 

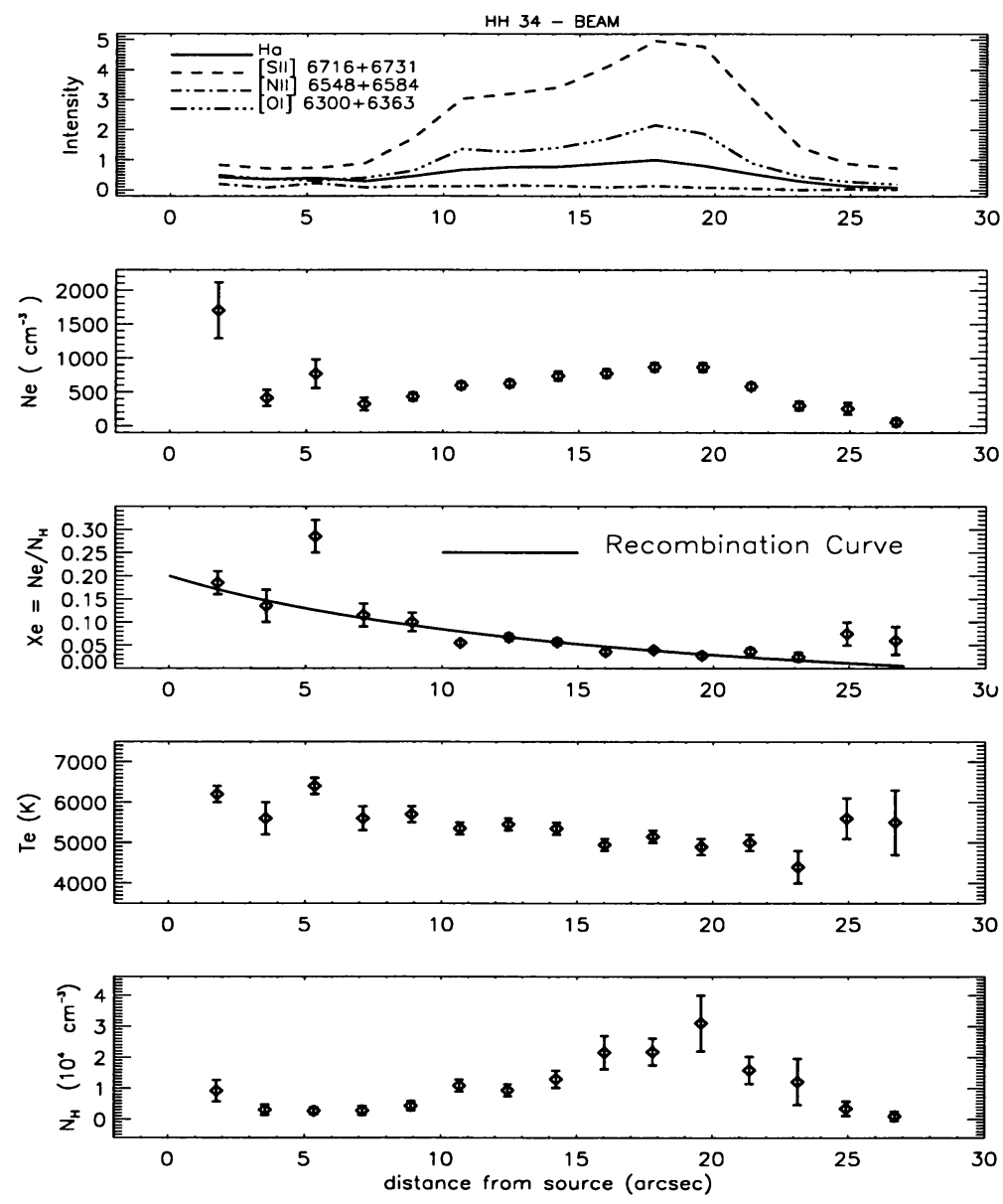

Figure 4. Physical conditions along the beam of the HH 34 jet. From top to bottom: a) intensity tracings normalized to $\mathrm{H} \alpha$ in the various lines $\mathrm{b}$ ) electron density derived form the [SII] lines ratio c) hydrogen ionization fraction $x_{e}$ and superimposed recombination curve (see text) d) average excitation temperature e) total hydrogen density $n_{H}=n_{e} / x_{e}$.

trating a) relative intensity tracings of the various lines integrated over the line widths, b) the electron density derived form the [SII] lines ratio, c) the hydrogen ionization fraction $x_{e}, \mathrm{~d}$ ) the average excitation temperature and e) the total hydrogen density $n_{H}$.

HH 34. Here the electron density is observed to increase progressively to knot J, after which it falls again (see Fig. 4). On the contrary, the ionization fraction decreases steadily from $x_{e}=0.2$ to about 0.03 from the beginning to the end of the visible section. The jump in $x_{e}$ at position $z=5 .^{\prime \prime} 3$ is 
probably affected by a large measurement error due to the faintness of the lines. Disregarding this point, the ionization nicely follows a recombination curve calculated assuming that on average the gas parcels flow in a set of nested cones, whose shape is specified by the initial jet radius and by a constant opening angle (see BE97 for details). Our best-fit curve turns out to have a negative opening angle $\theta=-1^{\circ} 4$, i.e., the jet is slightly converging, in accordance with the behavior of $n_{e}$. The average excitation temperature mildly decreases along the flow from about $6000 \mathrm{~K}$ to about $4300 \mathrm{~K}$. Finally, the total density profile shows a behavior similar to that of the electron density: after a slight decline prior to $z=5^{\prime \prime}$ the total density increases from $4-500 \mathrm{~cm}^{-3}$ up to $310^{4} \mathrm{~cm}^{-3}$ near knot $\mathrm{J}$, then it decreases again. From the total density a mean value of $n_{H} \sim 110^{4} \mathrm{~cm}^{-3}$ along the jet can be deduced, in accord with BCO95.

HH 24C. In this jet $n_{e}$ decreases on average along the flow, but shows two peaks at the position of the two brightest knots, with $n_{e} \sim 800 \mathrm{~cm}^{-3}$ and $\sim 400 \mathrm{~cm}^{-3}$ respectively (see Fig. 5). The ionization fraction oscillates around a mean value of $\sim 0.33$, with a slight increase in the fainter section at the middle of the flow, where the gas is apparently re-ionized: contrary to $\mathrm{HH} 34$ the ionization data points are better fit by two different recombination curves, the first one appropriate for a slightly converging jet section described by an angle $-2^{\circ}$, while the second one corresponds to flow surfaces opening by a small angle $1^{\circ}$. The average excitation temperature varies around 5500 to $6000 \mathrm{~K}$, increasing slightly toward the end of the bright beam section. The average total density is about $110^{3} \mathrm{~cm}^{-3}$. Assuming a jet velocity of $400 \mathrm{~km} \mathrm{~s}^{-1}$ the average mass loss rate obtained for this jet is $\dot{M}=6.810^{-8} \mathrm{M}_{\odot} \mathrm{yr}^{-1}$ while the momentum transfer rate is $\dot{P}=2.710^{-5}$ $\mathrm{M}_{\odot} \mathrm{yr}^{-1} \mathrm{~km} \mathrm{~s}^{-1}$.

$H H 24 E$. The presence of re-ionization episodes is even more evident in the HH $24 \mathrm{E}$ counterjet, where $x_{e}$ shows two pronounced jumps at positions $z=-10 . " 7$ and $z=-21 . " 3$, i.e. at the beginning of knot $\mathrm{E}$ and of the bright terminal condensation $\mathrm{HH} 24 \mathrm{~A}$, respectively. The ionization fraction decreases smoothly downstream of each jump, albeit along different recombination curves, that correspond to opening angles of $2^{\circ}, 1^{\circ}$, and $6^{\circ}$, respectively (with an assumed space velocity of $350 \mathrm{~km} \mathrm{~s}^{-1}$ ). On average, $x_{e}$ becomes progressively higher from one section to the next. The average excitation temperature also increases suddenly from 5100 to $6800 \mathrm{~K}$ at position $z=-10 . " 7$, while near knot A the increase is smoother and only of $1000 \mathrm{~K}$. The electron density is observed to markedly increase in knot A, while no apparent enhancement is seen near the first jump at $z=-10 .^{\prime \prime} 7$. The derived total density is higher in the first section of the flow. As a mean 

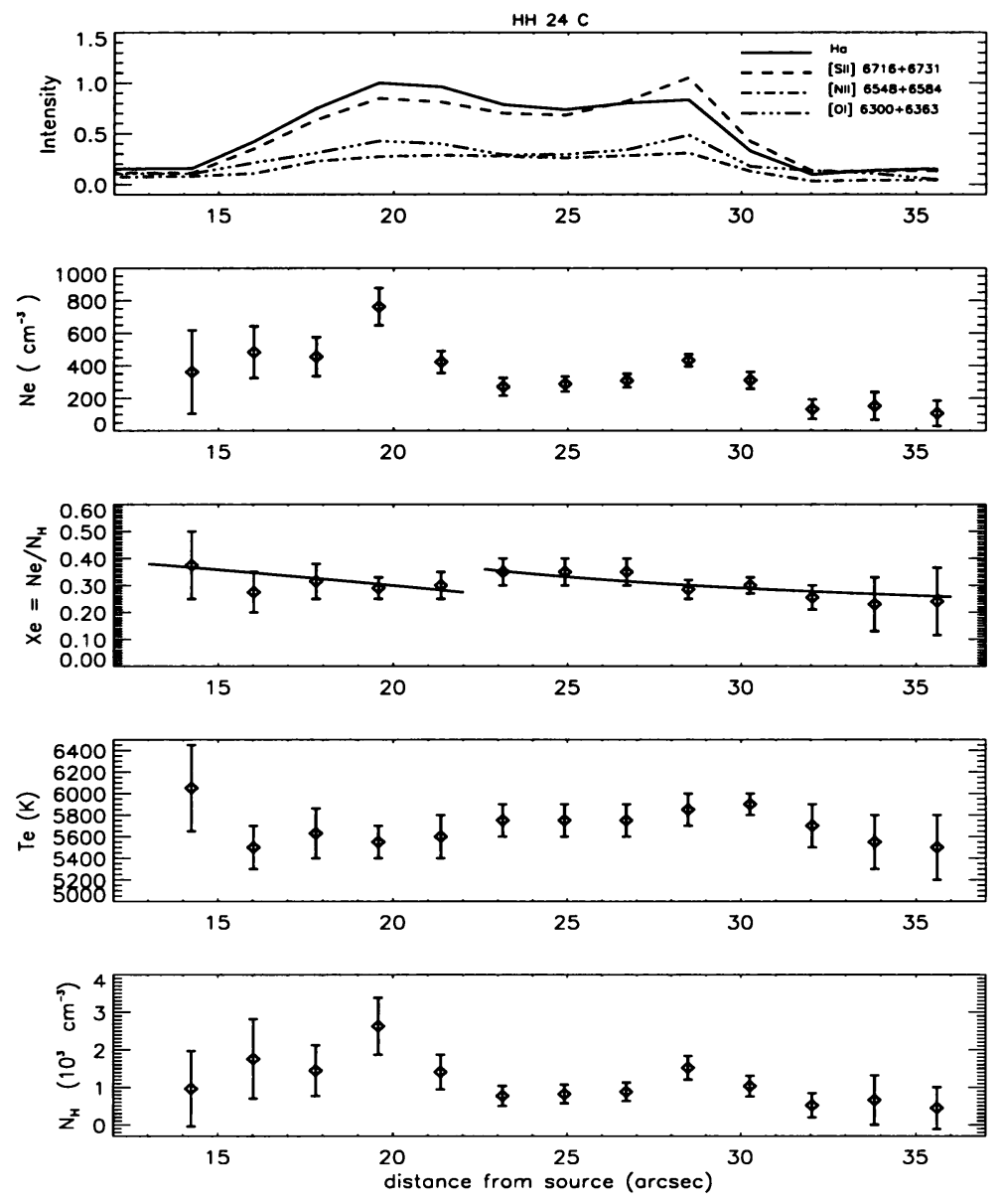

Figure 5. Physical conditions along the beam of the $\mathrm{HH} 24 \mathrm{C}$ jet. Panel description as in Fig. 4.

along the jet one can take $n_{H}=2.510^{3} \mathrm{~cm}^{-3}$, which yields $\dot{M}=6.110^{-8}$ $\mathrm{M}_{\odot} \mathrm{yr}^{-1}$ and $\dot{P} \approx 2.110^{-5} \mathrm{M}_{\odot} \mathrm{yr}^{-1} \mathrm{~km} \mathrm{~s}^{-1}$.

\section{Conclusions}

The BCO technique has been demonstrated to be a powerful tool for a model-independent investigation of the physical conditions in the beams of $\mathrm{HH}$ jets. A summary of the results is presented in the following.

- The gas in the beam is partially ionized; $x_{e}$ ranges between 0.05 and 0.4 , being higher for more excited and lighter jets. Both high temperatures and low densities, in fact, disfavour recombination. 

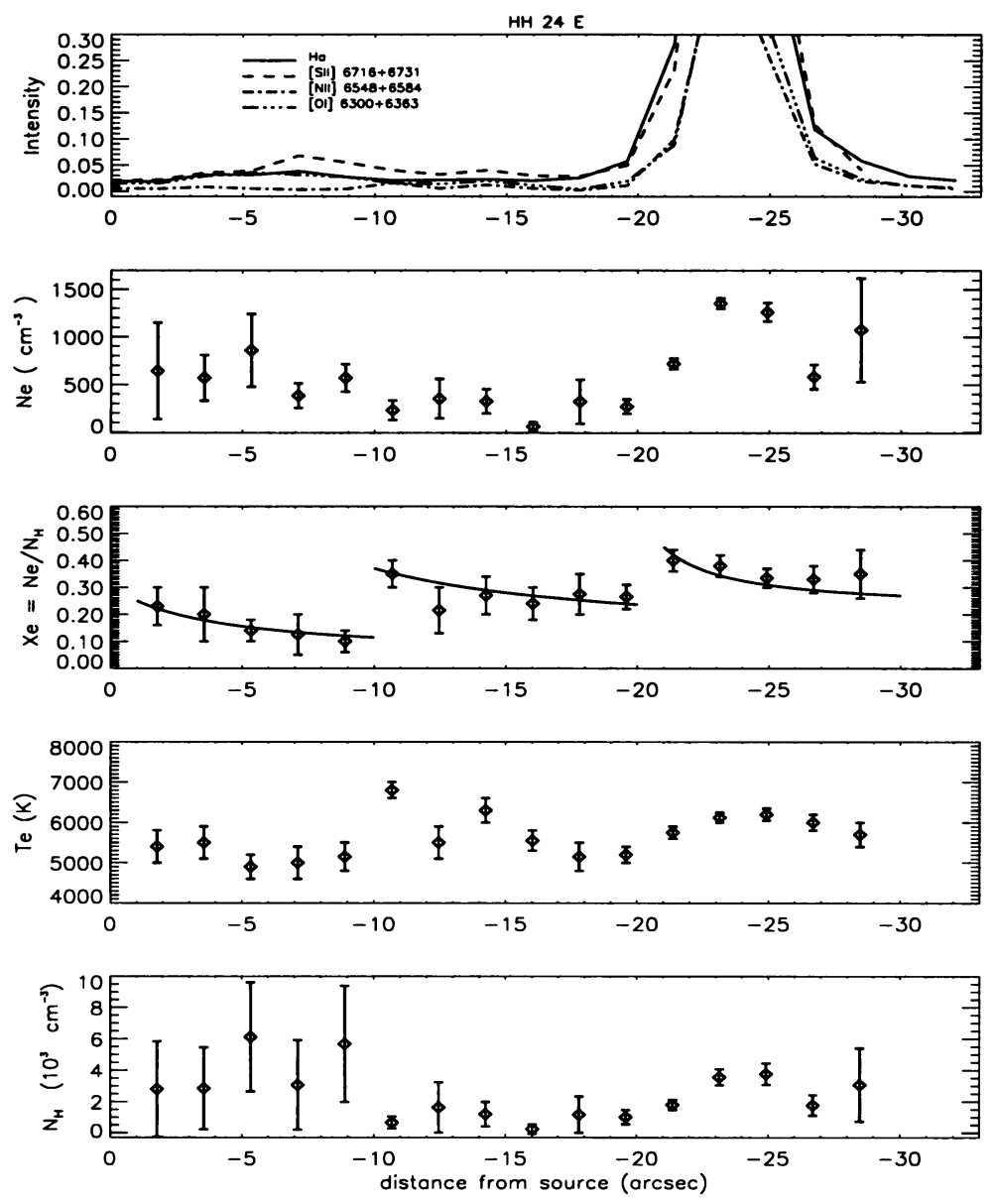

Figure 6. Physical conditions along the beam of the HH 24E jet. Panel description as in Fig. 4.

-The ionization fraction slowly decreases along the jet or along sections of it. As it was originally suggested in BCO95, the ionization state of the jet gas is probably produced in the acceleration region; then the gas slowly recombines traveling away from the source. If the shocks shaping the beam have $v_{s}<30-35 \mathrm{~km} \mathrm{~s}^{-1}$, they can only add a negligible contribution to the ambient ionization, as it apparently occurs in the optical outflow of RW Aur and in the HH 34 jet. The jumps in $x_{e}$ followed by slow decay observed in the $\mathrm{HH} 24 \mathrm{C} / \mathrm{E}$ flow are probably due to stronger internal shocks, that substantially re-ionize the gas.

- The excitation temperature varies between 4500 and $7000 \mathrm{~K}$. The limited spatial resolution of the investigated spectra does not allow to study 
in detail the post-shock cooling region and therefore the obtained values are averages over a large parcel of gas. Taking into account this constraint, the diagnostic results are not in contradiction with the prediction of shock models (see Hartigan et al. 1994, BE97).

- The average total hydrogen density $n_{H}=n_{e} / x_{e}$ ranges between about $10^{3}$ and a few $10^{4} \mathrm{~cm}^{-3}$. Average mass loss and momentum transfer rates calculated under the assumption of constant density over the jet section are of the order required to drive surrounding molecular outflows.

- Partial ionization should be taken into account in any jet model. Reliable calculations for beam shocks should consider that the fronts advance in a substantially ionized medium. In addition, partial ionization may introduce important differences in the modeling of magnetized jets, due to the effects introduced by collisions between charged particles and neutrals (see Bacciotti, Chiuderi and Pouquet 1997 for a discussion).

Acknowledgements: It is a pleasure to thank Alex Raga, Jochen Eislöffel and Tom Ray, without the help of whom the work presented in this paper would not have been possible.

\section{References}

Bacciotti, F., Chiuderi, C., and Oliva, E., 1995, A\&A, 296, 185 (BCO95)

Bacciotti, F., Hirth, G. and Natta, A., 1996, A\&A, 310, 309

Bacciotti, F., Chiuderi, C. and Pouquet, A., 1997, Ap J, 478, 594

Bacciotti, F., and Eislöffel, J., 1997, A\&A, submitted (BE97)

Bodo, G., Massaglia, S., Ferrari, A., and Trussoni, E., 1994, A\&A, 283, 655

Cabrit, S., Edwards, S., Strom, S., and Strom, K., 1990, ApJ 354, 687

Chernin, L., and Masson, C., 1995, ApJ, 455, 182

Edwards, S., Cabrit, S., Strom, S., Heyer, I., Strom, K., and Anderson, E., 1987, ApJ 321,473

Eislöffel, J., and Mundt, R., 1992, A\&A 263, 292

Hartigan, P., Morse, J., and Raymond, J., 1994, ApJ, 436, 125

Königl, A., and Ruden, S.P., 1993, in Protostar and Planets III, Levy G. and Lumine, J. eds. Tucson: University of Arizona press, 641.

Mundt, R., Ray, T.P., and Raga, A. C., 1991, A\&A 252, 740.

Osterbrock, D.E., 1989, Astrophysics of Gaseous Nebulae and Active Galactic Nuclei, University Science Books, Mill Valley, CA.

Raga, A.C., 1992, private communication.

Raga, A.C., and Kofman, L., 1992, ApJ 386, 222

Ray, T.P., Mundt, R., Dyson, J.E., Falle, S.A.E.G. and Raga, A.C., 1996, ApJ, 468, L103

Reipurth, B, 1989, Nature, 340, 42

Reipurth, B., Bally, J., Graham, J.A., Lane, A.P., and Zealey, W.J., 1986, A\&A 164, 51

Stone, J.M., and Norman, M.L., 1993, ApJ 413, 198 